\title{
PRODUCTION AND TESTING CONSIDERATIONS FOR CESR-C WIGGLER MAGNETS *
}

\author{
D. Rice ${ }^{\dagger}$, S. Chapman, R. Gallagher, Y. He, J. Kandaswamy, V. Medjidzade, A. Mikhailichenko, \\ N. Mistry, T. Moore, S. Richichi, K. Smolenski, A.B. Temnykh, W. Trask, \\ Laboratory for Elementary-Particle Physics, Cornell University, Ithaca, NY, USA \\ E. Smith, Laboratory of Atomic and Solid State Physics, Cornell University
}

Abstract

After construction of a prototype unit, five additional wiggler magnets for the CESR-c conversion have been completed at a rate exceeding one per month. These $2.1 \mathrm{~T}$ superferric magnets are built and assembled primarily in house with a minimal staff. We describe the general design and fabrication methods for these magnets. An additional 10 magnets will be constructed to complete the complement in the ring plus two spare units.

\section{INTRODUCTION}

The CESR-c conversion [1] at Cornell University's Laboratory for Elementary-Particle Physics will increase the world data set in the 3 to $5 \mathrm{GeV}$ c.m. range by factors of 20 to 100 in the next 4 years. Achieving luminosities well above $10^{32} \mathrm{~cm}^{-2}-\mathrm{sec}^{-1}$ in a machine optimized for $10.6 \mathrm{GeV}$ c.m. energy however requires significant modifications. In addition to the lower beam rigidity, the loss of a factor of 20 in radiation damping at the lower energy affects injection, single beam stability, and luminosity performance.

\section{Wiggler Design Requirements}

Restoration of this radiation damping is the dominant hardware task in the CESR-c conversion program. Over $15 \mathrm{~m}$ of 2.1 Tesla wiggler magnets generate the radiation to accomplish the damping. Details of the magnetic design may be found in reference [2]. The peak field is chosen as high as possible given limits on acceptable energy spread in the beam, which scales as $\sqrt{B}$.

Field quality requirements are high because of their dominant role in the CESR guide field. In the CESR-c layout the wigglers produce $90 \%$ of the synchrotron radiation in the ring. Furthermore the different horizontal closed orbits (up to $\pm 20 \mathrm{~mm}$ ) of the electrons and positrons used to separate the beams at parasitic crossings causes them to pass through the wigglers far off the central axis with opposite displacements. Investigations with tracking codes have shown that the field at the (longitudinal) center of each wiggler pole must drop off no more than $0.3 \%$ at $\pm 45 \mathrm{~mm}$ (the vacuum chamber aperture). [3,4] A $\pm 25 \mathrm{~mm}$ vertical beam-stay-clear mandates an unusually large gap between poles.

The requirements above quickly led us to choose superferric technology over conventional copper-ferric (too much power) or permanent magnet (precluded by large pole spacing).

\footnotetext{
* Work supported by the U.S. National Science Foundation

†dhr1@cornell.edu
}

\section{WIGGLER DESIGN}

\section{Principle Design Features}

Having chosen the $2.1 \mathrm{~T}$ peak field as described above, the period of the wiggler was set at $40 \mathrm{~cm}$ as a compromise between the inherent vertical non-linearities (increasing as $1 / \lambda^{2}$ where $\lambda$ is the pole period) and the field roll-off induced non-linearities that increase as $\lambda^{2}$. The length was chosen as $1.725 \mathrm{~m}$ flange-to-flange $(1.3 \mathrm{~m}$ active length) to optimize use of available space.

We spent considerable time deciding between odd and even pole configurations. While the arguments are too detailed to cover here, the greater flexibility in operating current (field quality) provided by the even pole arrangement carried the decision in the end (units 1 and 2 are 7-pole, units 3 and higher are 8-pole). Four fulllength $(20 \mathrm{~cm}$ long) inner poles are bordered by two $15 \mathrm{~cm}$ poles, followed in turn by end poles $10 \mathrm{~cm}$ long and with reduced strength to give $1 / 4$ bend angle relative to the central poles. This configuration gives zero net angle and displacement, and minimizes the asymmetry in trajectory through the wiggler.

The cold mass is enclosed in two separate volumes of liquid helium, joined by several jumper tubes with bellows. This cold mass is shielded from the warm beam pipe and cylindrical enclosure by liquid nitrogen cooled aluminum sheets. A companion paper [5] in this conference describes the cryostat in detail.

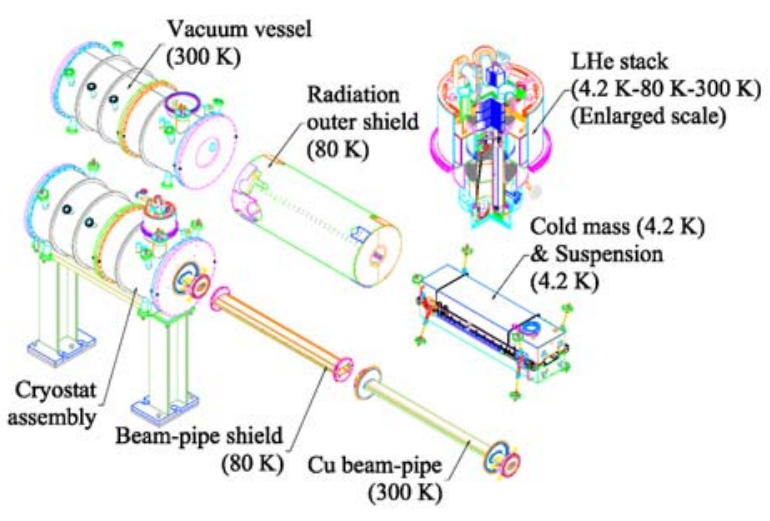

Figure 1: CESR-c wiggler components

Cryogens are provided from a $150 \mathrm{~m}$ long transfer line tapping into a preexisting system for the CESR superconducting RF cavities. Local PID controllers 
regulate cryostat $\mathrm{He}$ gas pressure and $\mathrm{LHe}$ and $\mathrm{LN}_{2}$ liquid levels. Each wiggler has its own main and trim (end poles) power supplies.

\section{MANUFACTURING PLAN}

For both scheduling and cost considerations, most of the fabrication and all assembly and testing are done in house. Outside work included:

- Machining and plating (where appropriate) of pole pieces, large iron and stainless-steel pieces, and several small pieces required in large quantities.

- Fabrication and leak checking of the cryostat outer jacket with all flanges.

- Fabrication of rolled or formed Al 77k shields.

- $\mathrm{Cu}$ extrusions for the beam line vacuum chamber.

\section{Manufacturing Components}

The component tasks of manufacturing the wigglers are easily broken into several parts that can be performed in a pipelined manner. These are described individually.

1) Coil winding: Coils are wound directly on individual machined and plated low carbon iron pole pieces. The main poles require 660 turns of $0.75 \mathrm{~mm}$ Formvar insulated $\mathrm{Nb}$ - $\mathrm{Ti}$ wire with $80 \mu \mathrm{m}$ filament diameter and 1.35:1 Cu:SC ratio. The wire is wet wound with Epotek T905 ${ }^{\mathrm{TM}}$ epoxy, stopping every five layers and clamping with shim blocks to maintain uniform dimensions - one hour is sufficient to set the wires in place. After a couple of weeks training, one winder can complete one main pole per day, or a trim pole (two windings) in two days.

2a) Placement and preloading of poles: The finished poles are placed on a $70 \mathrm{~mm}$ thick "yoke plate" that serves as flux return and support. Since the windings of adjacent poles experience large magnetic forces under operation, the coil assembly must be preloaded with about 16 tons of force ( $\sim 40 \mathrm{MPa}$ pressure on coils) to prevent coil motion after cooldown and the accompanying shrinkage of material. Because of small variations in final coil package dimensions, the gaps between coils must be filled with custom fitted shims to assure uniform pressure.

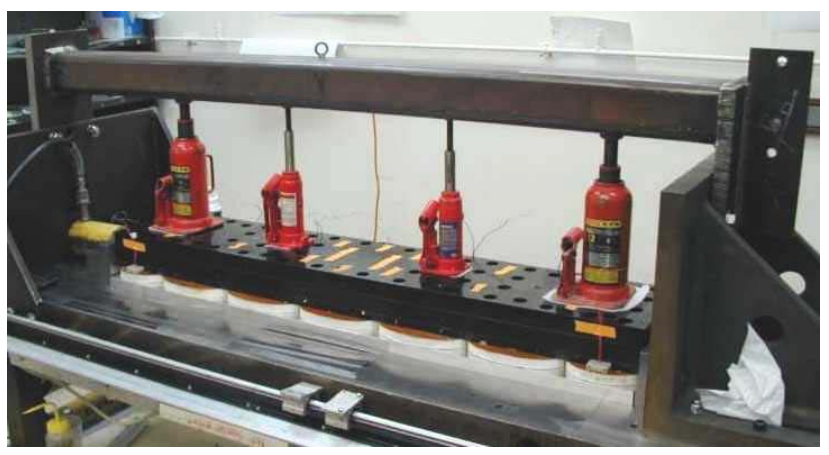

Figure 2: Magnet preload (yellow cylinder on the left)

Once the preload pressure is applied the pole pieces are pinned in place and endplates installed. After preload the wiring on the half-magnet is completed and checked. 2b) Cold mass enclosure: Next the assembly is placed on the stainless-steel (s-s) base plate and $6 \mathrm{~mm} \mathrm{~s}$-s plates welded on the sides and ends. A preliminary leak-check of the half cold mass assembly is done at this time since the connecting tubes cannot be rewelded once the connecting wires are installed in them. Then the top and bottom s-s plates (also $6 \mathrm{~mm}$ ) are welded in place. The two half cold masses are assembled around the beam pipe and inner heat shield, and leak checked.

3) Assembly in cryostat: Once assembled, the whole cold mass assembly is placed on rails ready for the cryostat and $77 \mathrm{~K}$ heat shield. A short section of the outer cryostat shell is first put in place to give access for installation of wiring and tubing. Once the stack (current lead and instrumentation feedthrough) and cryogen tubing connections are made up, the second shell section is installed and suspension adjusted to properly position the cold mass. Vacuum leak checks are performed before and after final welding of the end plates.

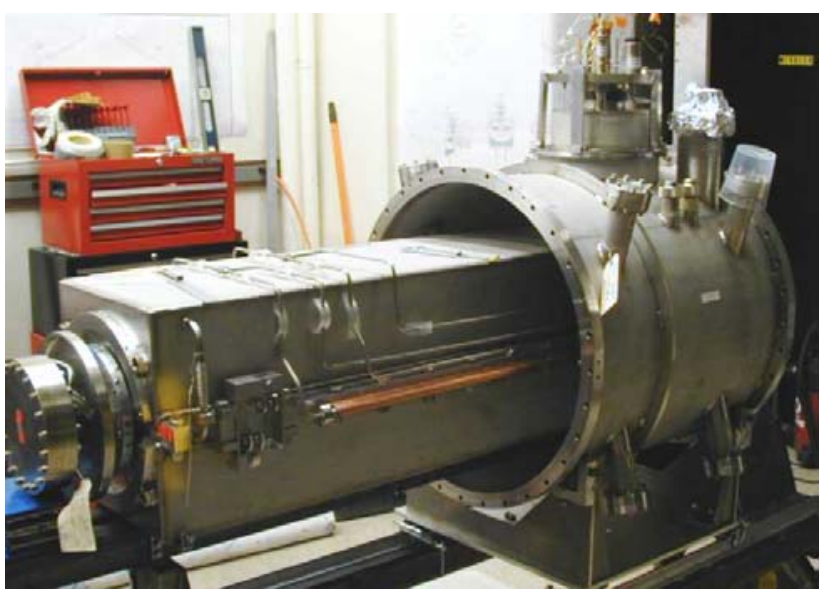

Figure 3: Cold mass in first cryostat section with stack

4) Training and field mapping: The finished wiggler is tested using LHe from a dewar (recovering the cold gas) and $\mathrm{LN}_{2}$ from the lab distribution system. During cool down cryogen usage and temperatures vs. time are recorded to check for irregularities. Magnet current is increased to full design level with one to three training quenches encountered in the first six wigglers. Field mapping uses both precision Hall probes and a flip coil for integral measurements. After field mapping the current is increased to $\sim 6 \%$ above maximum operating level. LHe use is recorded throughout the testing.

Alignment: During assembly the magnet iron is referenced to the cold mass enclosure, then the cold mass enclosure to survey fixtures on the end flanges of the cryostat using a total station (theodolite) instrument. The final uncertainty in positioning of the magnet in the accelerator reference system is estimated to be $\pm 1 / 2 \mathrm{~mm}$ in position and $\pm 1 / 2 \mathrm{mr}$ roll.

\section{Manufacturing Scheduling}

In a continuous production mode, each of the tasks, 1-4, above can be done overlapping with the others, though in practice our limited resources are often shared between 
two or more, reducing the pipelining efficiency. Figure 4 shows how these tasks are scheduled for several wigglers.

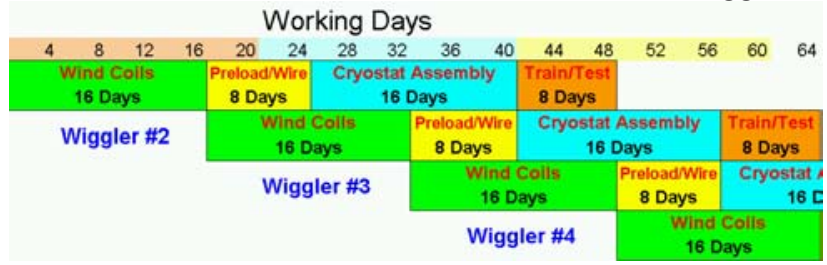

Figure 4: Pipelined manufacturing schedule

The schedule above is based on our experience with the last three wigglers (units 4-6) built for installation in the storage ring during Spring, 2003.

In addition, there are several asynchronous tasks such as beam line chamber fabrication and testing, assembling $77 \mathrm{~K}$ heat shields, constructing wiring harness, bending tubing, etc. Several other operations are hidden in the schedule above - for example, we have two coil winders who spend $2 / 3$ time actually winding, and the balance doing preparation \& cleanup work and occasional work on other projects.

During the production period (one wiggler every three weeks) internal resources averaged about five full time equivalent (FTE) senior technical and supervisory people and 13 FTE technical support people. The cost for parts and outside machining and fabrication was about $\$ 80 \mathrm{k}$ per wiggler unit.

\section{TESTING PROGRAM}

\section{Evolution of the Testing Program}

The testing program evolved significantly during the manufacture of the first six wiggler units. Initially we cold tested most of the individual pole windings before assembly on to the yoke plate. Before welding the LHe enclosure around the half-magnets, after which replacing a defective pole becomes much more difficult, we tested the entire magnet in a vertical dewar. Finally we tested the completed unit in its permanent cryostat.

The single pole cold tests, even when we made fixtures to test two at once, were labor intensive and required significant quantities of LHe. We quickly reduced this program to testing one or two poles per magnet, then only if the coil winders or warm test (see below) suggested some problem or change in technique. No poles failed this test once the winding technique was established.

The vertical test was abandoned after wigglers \#2 and \#3 showed no failures. (The prototype wiggler, \#1, developed a ground fault in the final horizontal test; subsequently we improved the insulation around the coil.) Dropping this test not only saves a week in the schedule and about $\$ 3,000$ of LHe, but not having the coils saturated with helium before assembly in the cold mass enclosure dramatically improves the sensitivity of the leak check process.

\section{Final Testing Program}

During coil production (before the individual cold tests were cut back) a warm test of individual poles was developed that proved effective in finding shorted turns and turn count errors. A pole is passed under a long, narrow pickup coil in $1 \mathrm{~cm}$ steps. The change in integrated voltage from the coil between zero and $1 \mathrm{amp}$ current is compared with a reference pole. Differences equivalent to 0.2 turns are readily visible, as are displacements in winding location of $20-30 \mu \mathrm{m}$.

This warm test, combined with the vigilance of the coil winders, has resulted in $100 \%$ good poles being installed in the wigglers with no further tests. Insulation testing is of course done at several stages of assembly - starting with 1000 volt tests of individual coils, being reduced to 500 volts after final assembly of the wiggler. Wiring continuity checks are also performed at several points.

The last three wigglers produced were cooled to LHe temperatures only after complete assembly into the cryostat. The time to replace a pole at this stage is approximately two weeks (but an additional penalty is disruption of production). So far this procedure has been effective. The measurements during this final test were briefly described above. Other than a small skew quadrupole component in the first two (7 pole) wigglers [6], the measured field has met all specifications.

\section{SUMMARY \& ACKNOWLEDGEMENTS}

The CESR-c wiggler design has proven to be robust and lends itself easily to moderate scale production. The field quality meets specifications and is very reproducible. We have developed an effective testing program that works well with the aggressive production schedule.

We gratefully acknowledge the talented technical support staff at LEPP. In particular we thank machine shop supervisors Chuck Firenze and John Kaminski for their suggestions to optimize the design for production and tireless work with outside machine shops.

\section{REFERENCES}

[1] D. Rice, "CESR-c - A Frontier Machine for QCD and Weak Decay Physics in the Charm Region," EPAC'02, Paris, June 2002, p. 428

[2] A. Mikhailichenko, "Optimized Wiggler Magnet for CESR,” PAC'01, Chicago, June 2001, p. 3648

[3] J. Crittenden et al., "Design Considerations for the CESR-c Wiggler Magnets," PAC'03, Portland, May 2003, WPAE009

[4] D. Sagan, "A Symplectic Model for Wigglers," PAC'03, Portland, May 2003, MPPG006

[5] Y. He et al., "Design and Operation of the Cryostat for the CESR-c Superconducting Wiggler Magnets," PAC'03, Portland, May 2003, WPPE037

[6] A. Temnykh, "Vibrating Wire and Flipping Coil Magnetic Measurement of a CESR-c 7-pole Wiggler Magnet,' PAC'03, Portland, May 2003, MPPG007 